\title{
Stimulus-Specific Adaptation in the Inferior Colliculus of the Anesthetized Rat
}

\author{
Manuel S. Malmierca, ${ }^{1,2}$ Salvatore Cristaudo, ${ }^{1}$ David Pérez-González, ${ }^{1,3}$ and Ellen Covey ${ }^{1,3}$ \\ ${ }^{1}$ Auditory Neurophysiology Unit, Institute of Neuroscience of Castilla y León, University of Salamanca, 37007 Salamanca, Spain, ${ }^{2}$ Department of Cell \\ Biology and Pathology, Faculty of Medicine, University of Salamanca, 37007 Salamanca, Spain, and ${ }^{3}$ Department of Psychology, University of Washington, \\ Seattle, Washington 98195
}

To identify sounds as novel, there must be some neural representation of commonly occurring sounds. Stimulus-specific adaptation (SSA) is a reduction in neural response to a repeated sound. Previous studies using an oddball stimulus paradigm have shown that SSA occurs at the cortex, but this study demonstrates that neurons in the inferior colliculus (IC) also show strong SSA using this paradigm. The majority (66\%) of IC neurons showed some degree of SSA. Approximately $18 \%$ of neurons showed near-complete SSA. Neurons with SSA were found throughout the IC. Responses of IC neurons were reduced mainly during the onset component of the response, and latency was shorter in response to the oddball stimulus than to the standard. Neurons with near-complete SSA were broadly tuned to frequency, suggesting a high degree of convergence. Thus, some of the mechanisms that may underlie novelty detection and behavioral habituation to common sounds are already well developed at the midbrain.

\section{Introduction}

The world is full of sounds that are common and repetitive. To attend to all of these sounds all of the time would be maladaptive. At the same time, however, it is crucial to attend to and evaluate novel sounds because these could signify danger or other circumstances that require an immediate behavioral response.

Most previous studies have assumed that adaptation to repetitive sounds and detection of novel sounds is a function of the auditory cortex (AC) (Csépe et al., 1987a,b; Näätänen, 1992; Tiitinen et al., 1994; Schönwiesner et al., 2007) and does not involve subcortical processing. This assumption is largely because of the fact that novelty detection has traditionally been studied by noninvasive recording of event-related potentials (ERP), especially the mismatch negativity (MMN) waveform, which appears $\sim 150$ $\mathrm{ms}$ after stimulus onset in response to an uncommon, or oddball, stimulus (Näätänen et al., 1978, 1992). MMN studies have had a profound impact on our understanding of many cognitive phe-

\footnotetext{
Received Aug. 25, 2008; revised Feb. 8, 2009; accepted Feb. 14, 2009

This work was supported by grants from the Spanish Ministerio de Ciencia e Innovación (BFU2006-00572) and the Junta de Castilla y León (JCYL) (GR221 and SAN673/SA20/08) to M.S.M. and the National Institutes of Health (NIH)-National Institute on Deafness and Other Communication Disorders (NIDCD; R01 DC-00287) and the National Science Foundation Grant I0S-0719295 to E.C. Support for software development was provided by NIH-NIDCD Gran P30DC004661; S.C. was supported by a Ministerio de Educacion y Ciencia fellowship (AP2005-5166). M.S.M and E.C. designed the experiments, supervised the project, interpreted the data, and wrote this manuscript; S.C. performed experiments and collected and analyzed data; D.P.G. gave conceptual advice, developed analytical tools, and analyzed data. We thank Drs. Alan Palmer, Leonard Kitzes, Dan Sanes, and Adrian Rees for their critical comments on a previous version and two anonymous reviewers for their constructive criticisms. We also thank Brandon Warren for software support and Flora Antunes for her help on the three-dimensional reconstructions.

Correspondence should be addressed to Dr. Manuel S. Malmierca, Auditory Neurophysiology Unit, Institute of Neuroscience of Castilla y León, University of Salamanca. C/ Pintor Fernando Gallego, 1, 37007 Salamanca, Spain. E-mail:msm@usal.es.

D. Pérez-González's present address: Institute of Neuroscience, Newcastle University, Newcastle upon Tyne NE2 $4 \mathrm{HH}, \mathrm{UK}$.

D01:10.1523/JNEUROSCI.4153-08.2009

Copyright $\odot 2009$ Society for Neuroscience $\quad$ 0270-6474/09/295483-11\$15.00/0
}

nomena and served in studies of various clinical populations (e.g., schizophrenia, Alzheimer's disease). However, the ERP method does not provide good information about processing at subcortical levels, nor does it provide accurate information about the identity and location of the neuronal generator of MMN in the cortex (Näätänen and Michie, 1979; Deouell, 2007; Fritz et al., 2007).

Recent electrophysiological studies in animals, using an oddball stimulus paradigm similar to that used to evoke MMN in humans, have focused on the cortex and demonstrated that many AC neurons in the cat exhibit a reduced response to a stimulus that is presented repeatedly. This phenomenon is termed stimulus-specific adaptation (SSA) (Ulanovsky et al., 2003, 2004; Nelken and Ulanovsky, 2007). When a novel (oddball) sound is presented, these same neurons are released from SSA and respond vigorously to one or a few presentations of the new sound. Based on recordings from a few neurons in the auditory thalamus [the medial geniculate body (MGB)], Ulanovsky et al. (2003) concluded that SSA does not occur below the level of the cortex.

However, a recent study in the rat (Pérez-González et al., 2005) has shown that the main auditory midbrain nucleus, the inferior colliculus (IC), contains a population of neurons that respond selectively to novel stimuli. These novelty selective neurons appear to be a highly specialized population, but it is not known whether, or to what extent, neurons in the IC exhibit SSA of the type seen in cortical neurons. The stimulus presentation methods used by Pérez-González et al. (2005) were somewhat different from those used by Ulanovsky et al. (2003) to study AC responses, and were not well suited to demonstrate or quantify partial SSA. To examine responses of a large population of neurons across the IC and to provide a direct comparison with the studies in cat cortex, we implemented an oddball stimulus paradigm, similar to that used by Ulanovsky and colleagues, to record 
auditory responses from the rat IC. Not only did this method allow us to evaluate the conditions under which SSA is seen in the rat IC and compare them with those that elicit SSA in the cat cortex, it also provides an indirect comparison with studies of MMN in humans. A preliminary report has been presented previously (Malmierca et al., 2007).

\section{Materials and Methods}

Surgical procedures. Experiments were performed on 42 adult rats with body weights between 190 and $410 \mathrm{~g}$. All experiments were performed at the University of Salamanca with the approval of, and using methods conforming to the standards of, the University of Salamanca Animal Care Committee.

Surgical anesthesia was induced and maintained with urethane (1.5 $\mathrm{g} / \mathrm{kg}$, i.p.), with supplementary doses ( $0.5 \mathrm{~g} / \mathrm{kg}$, i.p.) given as needed. Urethane was chosen as an anesthetic because its effects on multiple aspects of neural activity including inhibition and spontaneous firing are known to be less than those of barbiturates and other anesthetic drugs (Hara and Harris, 2002). The trachea was cannulated, and atropine sulfate $(0.05 \mathrm{mg} / \mathrm{kg}$, s.c.) was administered to reduce bronchial secretions. Body temperature was maintained at $38^{\circ} \mathrm{C} \pm 1^{\circ} \mathrm{C}$. Details of surgical preparation were as described previously (Malmierca et al., 2003, 2005a,b, 2008; Hernández et al., 2005; Pérez-González et al., 2005, 2006). The animal was placed in a stereotaxic frame in which the ear bars were replaced by hollow specula that accommodated a sound delivery system.

Acoustic stimuli and electrophysiological recording. A craniotomy was performed to expose the cerebral cortex and the cerebellum overlying the IC. A tungsten electrode (1-2 M $\Omega$ ) (Merrill and Ainsworth, 1972) was lowered through the cortex and used to record extracellular single unit responses in the IC. Neuron location in the IC was based on stereotaxic coordinates, physiological criteria of tonotopicity, response properties, and histological verification using electrolytic lesions (5-10 $\mu \mathrm{A}$ for 5-10 s) to mark recording sites (Malmierca et al., 1993, 1995, 2003, 2008; LeBeau et al., 2001; Hernández et al., 2005; Pérez-González et al., 2005, 2006).

Stimuli were delivered through a sealed acoustic system (Rees, 1990, 1997) using two electrostatic loudspeakers (TDT-EC1) driven by two TDT-ED1 modules. Pure tone bursts were delivered to one or both ears under computer control using TDT System 2 (Tucker-Davis Technologies) hardware and custom software (Faure et al., 2003). Typically, tones were $75 \mathrm{~ms}$ duration with a $5 \mathrm{~ms}$ rise/fall time. Selected parameters were varied one at a time during testing. The electrode was advanced using a Burleigh microdrive. Action potentials were recorded with a BIOAMP amplifier (TDT), the $10 \times$ output of which was further amplified and bandpass-filtered (TDT PC1; $f_{\mathcal{c}}, 500 \mathrm{~Hz}$ and $3 \mathrm{kHz}$ ) before passing through a spike discriminator (TDT SD1). Spike times were logged on a computer by feeding the output of the spike discriminator into an event timer (TDT ET1) synchronized to a timing generator (TDT TG6). Stimulus generation and on-line data visualization were controlled with custom software. Spike times were displayed as dot rasters ordered by the acoustic parameter varied during testing. Search stimuli were pure tones or noise bursts.

To the extent possible, the approximate frequency tuning of the cell was audiovisually determined. The minimum threshold and best frequency $(\mathrm{BF})$ of the cell were obtained by an automated procedure with $2-5$ stimulus repetitions at each frequency and intensity step. The frequency response area (FRA), i.e., the combination of frequencies and intensities capable of evoking a response, was then obtained automatically using a randomized stimulus presentation paradigm and plotted using Excel (Microsoft) and SigmaPlot (Systat) software.

Stimulus presentation paradigms. For all neurons, stimuli were presented in an oddball paradigm similar to that used to record mismatch negativity responses in human studies (Näätänen, 1992) and more recently in the cat auditory cortex (Ulanovsky et al., 2003, 2004). Briefly, we presented two stimuli consisting of pure tones at two different frequencies $\left(f_{1}\right.$ and $\left.f_{2}\right)$, that elicited a similar firing rate at a level of $10-40 \mathrm{~dB}$ above threshold. Both frequencies were within the excitatory frequency response area previously determined for the neuron (Fig. $1 A$ ). We pre-

A Condition 1 $\begin{array}{cc}\square \square \square \square \square \square \square \square \square & f_{1} \square \\ f_{1}: 90 \%, f_{2}: 10 \% & f_{2} \square\end{array}$ Condition $2 \square \square \square \square \square \square \square \square \square \square$ $f_{1}: 10 \%, f_{2}: 90 \%$

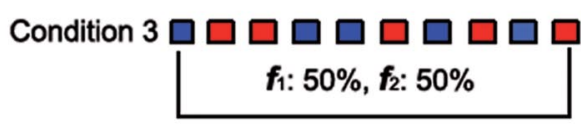

B
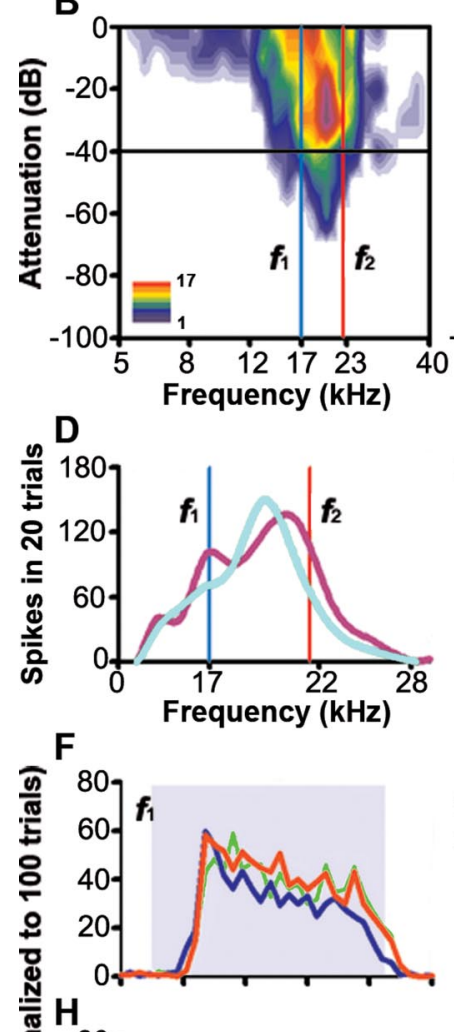

G

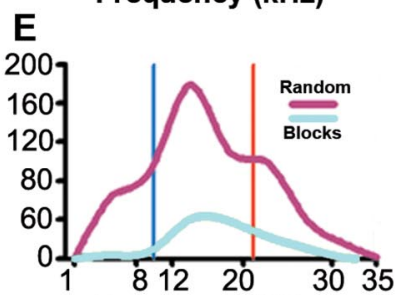

Frequency (kHz)

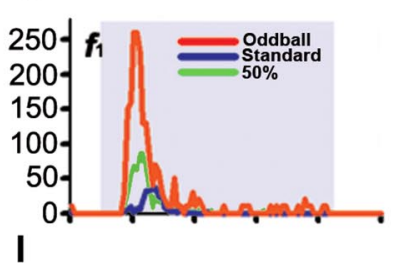

\section{1}

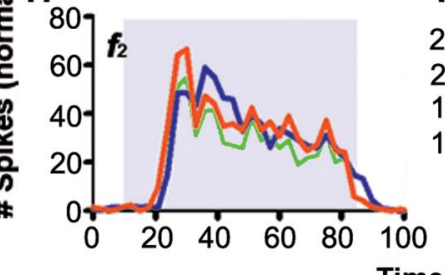

Time (ms)

Figure 1. Examples of the responses of two $\mathrm{IC}$ neurons to sounds presented in an oddball paradigm. $\boldsymbol{A}$, Sequences of pure tones at two different frequencies $\left(f_{1}, f_{2}\right)$ were presented, varying the probability of each of the frequencies. In Condition $1, f_{1}$ had $90 \%$ probability of occurring and $f_{2}$ a $10 \%$ probability. In Condition 2 the probabilities of $f_{1}$ and $f_{2}$ were reversed. Finally, in Condition 3 both frequencies occurred with equal (50\%) probability. In this and similar figures the high probability stimulus is referred to as standard, and the low probability one as the oddball. $\boldsymbol{B}$, FRA of a neuron that showed little adaptation; $\boldsymbol{C}$, FRA of a neuron that showed considerable SSA. The red and blue vertical lines on both FRAs show the two frequencies presented in the oddball paradigm and the black horizontal line shows the level. $\boldsymbol{D}$ and $\boldsymbol{E}$ show spike counts as a function of frequency for the same two neurons when frequencies were presented in random order (magenta line) or blocks of identical frequencies (light blue line). $\boldsymbol{F}$ and $\boldsymbol{H}$ show the normalized responses of the neuron on the left to tones presented using the oddball paradigm. The red trace is the response to the oddball stimulus (see $A, f_{1}$ in Condition 2 and $f_{2}$ in Condition 1); the blue trace is the response to the standard ( $f_{1}$ from Condition 1 and $f_{2}$ from Condition 2), and the green line is the response when both tones were presented with equal probability ( $f_{1}$ and $f_{2}$ from Condition 3 ). $G$ and $I$ show the responses of the neuron on the right, presented in the same format. In this and similar figures, the shaded background indicates the duration of the stimulus. 
A
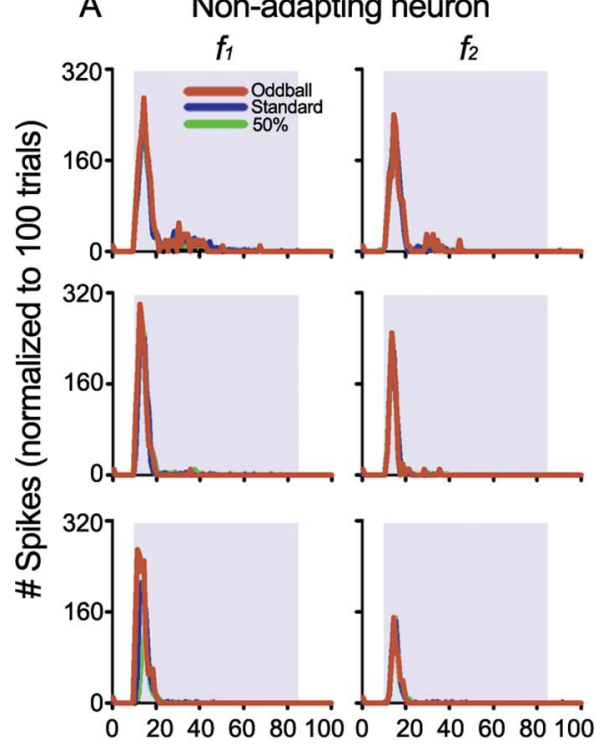

B
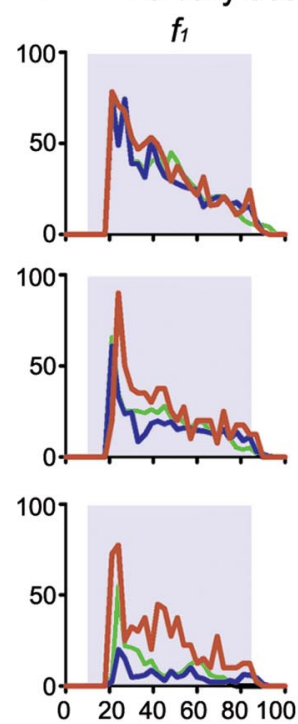

C Novelty neuron
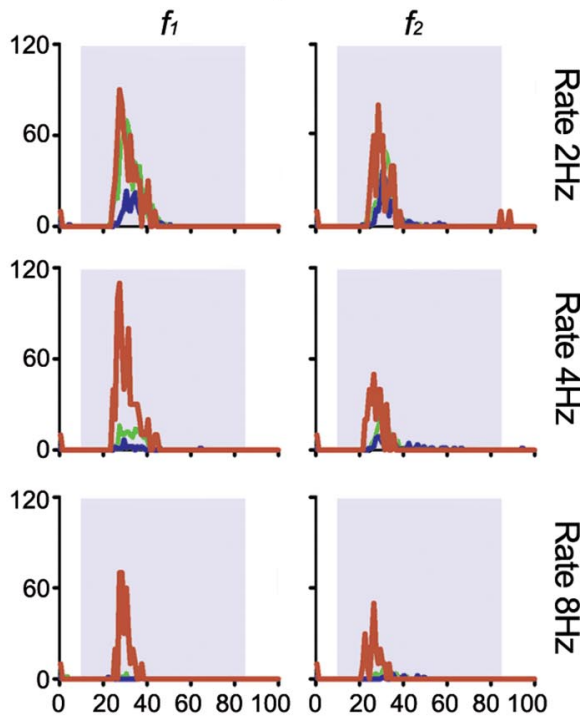

Time (ms)

Figure 2. Effect of stimulus repetition rate on neurons with different amounts of adaptation, using the oddball paradigm. $\boldsymbol{A}$, In a nonadapting neuron, the responses to the standard (blue traces) and oddball (red traces) stimuli are similar regardless of the repetition rate ( $2 \mathrm{~Hz}$, top row; $4 \mathrm{~Hz}$, middle row; $8 \mathrm{~Hz}$, bottom row). $\boldsymbol{B}$, Partially adapting neurons responded better to the oddball at the higher repetition rates. C, Novelty neurons responded much more strongly to the oddball than to the standard at all repetition rates tested. In all neurons that showed SSA, the response to the $50 \%$ probability condition was always smaller than that to oddball but larger than to the standard.

sented a train of 400 stimuli containing both frequencies in a probabilistic manner at a specific repetition rate. As shown in Figure $1 A$ (Condition 1 ), one frequency $\left(f_{1}\right)$ was presented as the standard (i.e., high probability within the sequence); interspersed randomly among the standards were the oddball stimuli (i.e., low probability) at the second frequency $\left(f_{2}\right)$. The custom software allowed us independently to vary the probability of the oddball stimulus and the amount by which it varied from the standard. After obtaining one data set, the relative probabilities of the two stimuli were reversed, with $f_{2}$ as the standard and $f_{1}$ as the oddball (Fig. $1 A$, Condition 2). As a control, $f_{1}$ and $f_{2}$ were presented with equal probability in a pseudorandom sequence (Fig. $1 A$, Condition 3 ). Each condition contained a total of 400 stimuli. The responses to the standard stimulus and oddball stimulus were normalized to account for the different number of presentations in each condition, because of the different probabilities.

The same paradigm was repeated varying the probability of the oddball $(10 \%, 30 \%)$ or the frequency contrast between the standard and oddball. The frequency contrasts were chosen to be as close as possible to values that have been used in other studies, i.e., $\Delta f=0.37,0.10,0.04$; where $\Delta f=\left(f_{2}-f_{1}\right) /\left(f_{2} \times f_{1}\right)^{1 / 2}$ is the normalized frequency difference (Ulanovsky et al., 2003, 2004). These values correspond to frequency ratios of $0.526,0.141$ and 0.057 octaves, respectively. We also tested the oddball paradigm at different repetition rates $(2 / \mathrm{s}, 4 / \mathrm{s}$, and $8 / \mathrm{s})$. It should be noted that the repetition rates tested here were generally faster than those used by Ulanovsky and colleagues in the AC, who used repetition rates between $0.25 / \mathrm{s}$ and $3 / \mathrm{s}$. These repetition rates were chosen based on the fact that on average, IC neurons can be driven at faster rates than neurons in the AC. To quantify the amount of SSA that occurred, we calculated two different forms of the SSA index using the method described by Ulanovsky et al. $(2003,2004)$. One was the frequency-specific index $\operatorname{SI}\left(f_{i}\right)$, where $i=1$ or 2 , defined for each frequency $f_{i}$ as $\operatorname{SI}\left(f_{i}\right)=$ $\left[\mathrm{d}\left(f_{i}\right)-\mathrm{s}\left(f_{i}\right)\right] /\left[\mathrm{d}\left(f_{i}\right)+\mathrm{s}\left(f_{i}\right)\right]$, where $\mathrm{d}\left(f_{i}\right)$ and $\mathrm{s}\left(f_{i}\right)$ are responses (as normalized spike counts) to frequency $f_{i}$ when it was oddball or standard, respectively. The other SSA index was the neuron-specific index (NSSI) defined as NSSI $=\left[\mathrm{d}\left(f_{1}\right)+\mathrm{d}\left(f_{2}\right)-\mathrm{s}\left(f_{1}\right)-\mathrm{s}\left(f_{2}\right)\right] /\left[\mathrm{d}\left(f_{1}\right)+\mathrm{d}\left(f_{2}\right)+\right.$ $\left.\mathrm{s}\left(f_{1}\right)+\mathrm{s}\left(f_{2}\right)\right]$, where $\mathrm{d}(f)$ and $\mathrm{s}(f)$ are responses to each frequency $f_{1}$ or $f_{2}$ when they were the oddball or standard stimuli, respectively.

These indices reflect the extent to which the response of a cell to the standard was suppressed and/or response to the oddball was enhanced.
The possible range of NSSI values is from -1 to +1 , being positive if the response to the oddball stimulus was greater, and negative if the response to the standard stimulus was greater. To thoroughly quantify the conditions that elicited SSA and novelty responses in a given neuron, the indices were analyzed as a function of probability ratio, contrast between oddball and standard, and repetition rate.

In 37 selected neurons, stimuli were presented using the same paradigm as in (Pérez-González et al., 2005). This paradigm included a "random" presentation mode, in which values of the variable parameter were randomized across trials, and a "block" mode, in which a block of 10-25 identical stimuli was presented, after which the parameter value was changed, and another block of trials was presented. This procedure was repeated at each step from the start value to the end value of the variable parameter. To estimate frequency discriminability by IC neurons, we performed a receiver operating characteristic (ROC) analysis based on the distributions of spike counts of each neuron. We computed the ROC curve for each pair of frequencies $\left(f_{1}, f_{2}\right)$, and the area under the curve was used as an estimation of the percentage of correct responses. For the $90 / 10 \%$ condition, we used the larger of the two percentage values calculated to facilitate direct comparisons with the data obtained by Ulanovsky et al. (2003).

Histological verification of recording sites. At the end of each experiment, the animal was given a lethal dose of sodium pentobarbital and perfused transcardially under deep surgical anesthesia with PBS $(0.5 \%$ $\mathrm{NaNO}_{3}$ in PBS) followed by fixative (a mixture of $1 \%$ paraformaldehyde and $1 \%$ glutaraldehyde in rat Ringer's solution). After fixation and dissection, the brain tissue was cryoprotected in $30 \%$ sucrose and sectioned on a freezing microtome in the transverse or sagittal planes into 40- to $50-\mu \mathrm{m}$-thick sections. Sections were stained with $0.1 \%$ thionin blue to facilitate identification of cytoarchitectural boundaries (Malmierca et al., 1993, 1995; Loftus et al., 2008). Recording sites were marked on standard sections from a rat brain atlas (Paxinos and Watson, 2005) and used to make three-dimensional reconstructions using Neurolucida software (Microbrightfield).

\section{Results}

The majority of IC neurons show SSA

We obtained data from 173 neurons throughout the entire extent of the IC while presenting stimuli in the oddball paradigm. The 
majority of IC neurons (66\%) responded more strongly to the oddball stimulus regardless of whether it was $f_{1}$ or $f_{2}$. Figure 1 compares typical examples from a neuron that did not show SSA (left column) and one that showed considerable SSA (right column). These two neurons were tested at a repetition rate of $4 \mathrm{~Hz}$ using both the oddball paradigm (Fig. $1 A$ ) and the original method of Pérez-González et al. (2005). Figure 1, D and E, shows isointensity functions ( $30 \mathrm{~dB}$ above threshold, see $B$ and $C$, black horizontal line) comparing spike counts as a function of frequency when frequencies were randomized (random, magenta trace), and when they were presented in blocks of identical stimuli (blocks, light blue trace). The response of the nonadapting neuron (Fig. $1 D$ ) was approximately the same regardless of stimulus presentation mode, while the "novelty" neuron's response was considerably larger when stimuli were presented randomly than when they were presented in blocks (Fig. $1 E$ ). Figure $1 F-I$ compares responses of the same neurons to two frequencies, $f_{1}$ and $f_{2}$, using the oddball paradigm. For the nonadapting neuron (Fig. $1 F, H)(\Delta f=0.76)$, the response to each of the two frequencies was about the same regardless of whether it was the standard (blue), oddball (red), or equal probability (green). For the "novelty" neuron (Fig. $1 G, I)(\Delta f=0.21)$, the response to the oddball was always significantly greater, regardless of which frequency was the oddball.

Although Figure 1 shows extreme cases, when all IC neurons were analyzed, we found that there was a continuum that covered the entire range from no SSA under any conditions to virtually complete SSA (close to maximal, NSSI $=1$ ) under all conditions. Figure 2 shows examples of three neurons, one with no SSA under any of the conditions tested $(A)$, one that showed variable degrees of SSA depending on stimulus repetition rate $(B)$, and one that showed SSA under all conditions tested $(C)$. For all three neurons, responses declined at high repetition rates, but the nonadapting neuron continued to respond equally to both the standard and oddball stimuli even at the highest repetition rate. The partially adapting neuron, however, responded about the same to both the standard and oddball stimuli at low repetition rates, but responded better to the oddball stimulus at higher repetition rates.

Repetition rate was not the only factor that determined how much adaptation (SSA) a neuron experienced. Figure 3 shows the responses of another neuron to different degrees of frequency contrast between $f_{1}$ and $f_{2}(A)$ and two different probabilities of the oddball stimulus $(B)$. When the frequency contrast was high ( $\Delta f=0.37$, row 1$)$, the response to the oddball was much greater than the response to the standard stimulus. As the frequency contrast decreased (rows 2 and 3), the response to the standard became stronger, while the response to the oddball remained approximately the same (Fig. $3 A$ ). When the probability of the oddball was increased from 10 to $30 \%$, the response to the oddball stimulus decreased slightly, but the response to the standard increased (Fig. 3B).

Figure 4 summarizes the pooled results from all of the 173 IC neurons in this study. As seen in the data described above for individual neurons, the degree of SSA and the response to the oddball stimulus depended on the stimulus repetition rate, the frequency contrast between $f_{1}$ and $f_{2}$, and the probability of occurrence of the oddball. Across the population, there was very little SSA at a contrast of $\Delta f=0.04$, which corresponds to 0.057 octaves, but considerable SSA at both of the higher values tested. Although the response to the standard stimulus decreased progressively as repetition rate increased, the largest difference between standard and oddball was at a stimulus repetition rate of

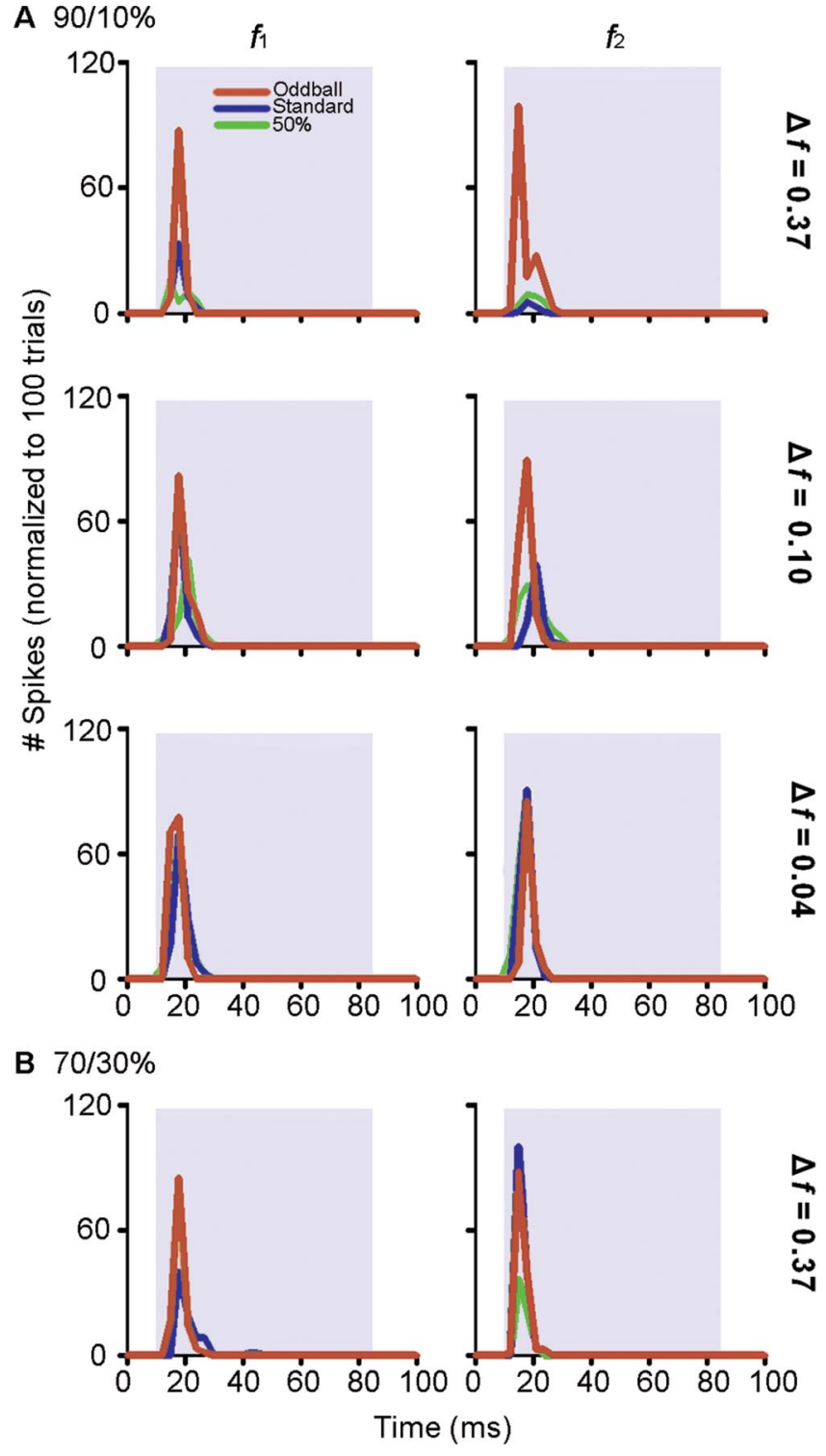

Figure 3. Effect of frequency contrast ( $\Delta f$, see Results for details) and probability on a single neuron in the IC when stimuli were presented using the oddball paradigm. $A, A n$ increase in contrast between the two frequencies $\left(f_{1}, f_{2}\right)$ resulted in an increase in the difference between the response to the standard and the response to the oddball. This difference was primarily caused by a reduced response to the standard as well as to an augmented response to the oddball. In this experiment, $f_{1}$ was held constant at $4.3 \mathrm{kHz}$ while $f_{2}$ was systematically changed to obtain the required $\Delta f$, i.e., $6.3 \mathrm{kHz}, 3.8 \mathrm{kHz}$, and $4.5 \mathrm{kHz}$ for $\Delta f=0.37,0.10$, and 0.04 , respectively. The top three rows $(\boldsymbol{A})$ show data obtained when the probability of the oddball was $10 \%$. $B$, When it was increased to $30 \%$, the difference between the standard and the oddball was reduced $(\Delta f=0.37)$.

4/s. As shown for individual neurons, SSA was greatest when the probability of the oddball was lowest. It is interesting to note that, under all conditions, the largest difference between standard and oddball responses [population difference signal (DS)], was in the onset component of the response, up to $20 \mathrm{~ms}$ after stimulus onset (Fig. 4, DS, green traces; see also Table 1).

Comparing the SSA-indices across different conditions allowed us to quantify the strength of adaptation. Figure 5 shows scatter plots of the frequency-specific SI for $f_{1}$ versus $f_{2}$ at different frequency contrast values, repetition rates, and oddball probabilities. Most values are positive across the entire range of conditions, with the majority of values lying in the upper right quad- 
A $90 / 10 \%$

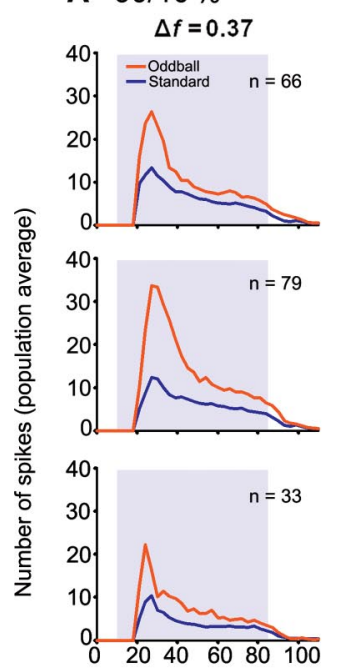

DS
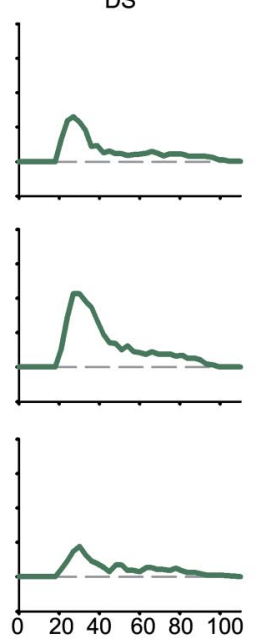

B $70 / 30 \%$
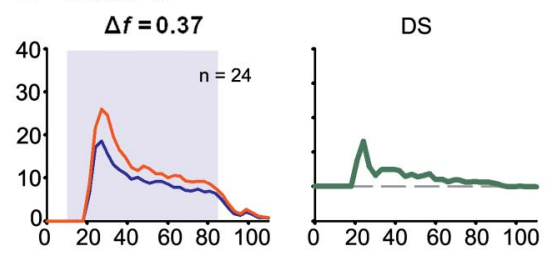

$\Delta f=0.10$
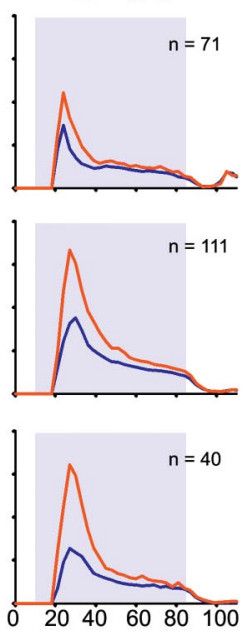

$\Delta f=0.10$

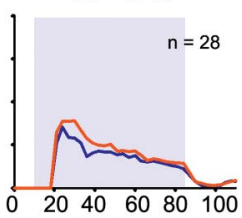

DS
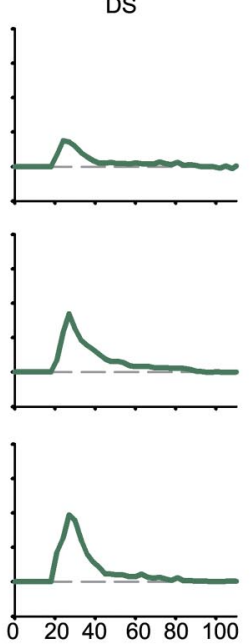

DS

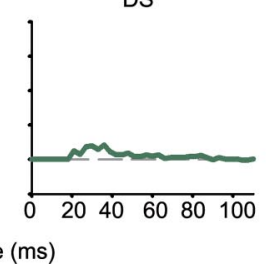

$\Delta f=0.04$
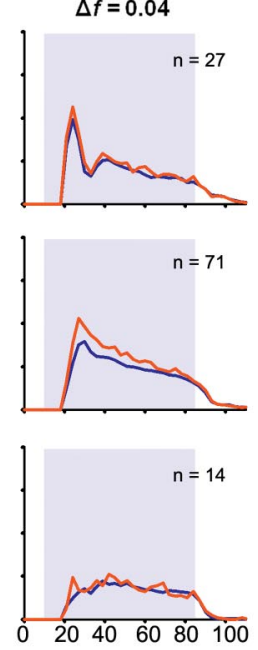

$\Delta f=0.04$

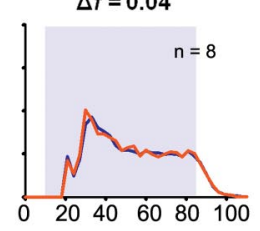

DS
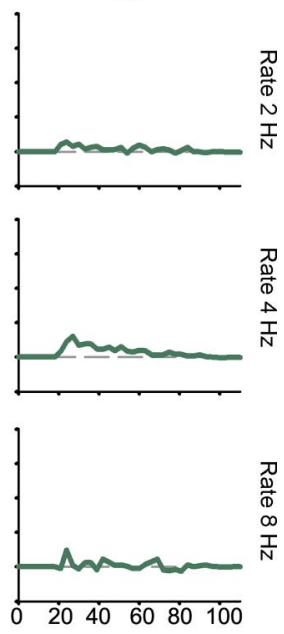

DS

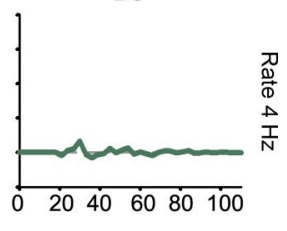

Figure 4. $\quad \boldsymbol{A}, \boldsymbol{B}$, Average effect of stimulus probability $(\boldsymbol{A}, 90 / 10 \% ; \boldsymbol{B}, 70 / 30 \%)$, frequency contrast (columns) and stimulus repetition rate (rows) across the population of IC neurons The peristimulus histograms (PSTH) for the standard and oddball stimuli were normalized to 100 trials to account for the different probabilities and averaged across the entire sample of neurons recorded from the IC. The number of neurons for each condition is indicated on each panel. The corresponding DS (difference signal) shown to the right of each PSTH represents the difference between the population response to the oddball and standard stimuli. DS is larger for larger frequency contrasts, and lower oddball probabilities. It reaches a plateau at a stimulus repetition rate equal to or larger than $4 \mathrm{~Hz}$.

Table 1. Average population DS latency (in ms)

\begin{tabular}{rlll}
\hline & $\Delta f=0.37$ & $\Delta f=0.1$ & $\Delta f=0.04$ \\
\hline $90 / 10 \%$ & & & \\
$2 \mathrm{~Hz}$ & 17 & 14 & 14 \\
$4 \mathrm{~Hz}$ & 19 & 17 & 17 \\
$8 \mathrm{~Hz}$ & 14 & 17 & 14 \\
$70 / 30 \%$ & & & 20 \\
$4 \mathrm{~Hz}$ & 20 & 26 & 20 \\
\hline
\end{tabular}

Compare to Figure 4.

rant of the graphs, distributed more or less continuously over the range from 0 to +1 . However, this relationship breaks down at the smallest frequency contrast and oddball probability values, where many neurons failed to respond at all. It is worth noting that at the largest two frequency contrast values, there is a cluster of neurons in the upper right corner, consisting of those that expressed a high degree of novelty selectivity. The distribution of neuron-specific SI (see supplemental Figs. 1, 2, available at www.jneurosci.org as supplemental material) also demonstrates that, under most conditions (different frequency contrast values, repetition rates, and oddball probabilities), the SI values are skewed to the positive side (Table 2). Only at the lowest frequency contrast and highest oddball probability were values clustered around zero (Fig. 5A,B).

Because of the large number of stimulus repetitions under each set of conditions, not all neurons were tested with all conditions. However, for 152 neurons that we were able to hold long enough to record more than three conditions, we averaged the neuron-specific SSA-indices across conditions (see supplemental Fig. 1, available at www.jneurosci.org as supplemental material). These results show that $85 \%$ of values were positive, forming a continuous distribution all the way to +1 , which represents complete SSA. Only $11 \%$ of the neurons (16) had negative values, indicating that they responded better to the standard stimulus than to the oddball stimulus. However, with two exceptions (NSSI $=-0.42$ and -0.11 ), the negative values were all very close to zero (range, -0.07 to 0 ), and were not statistically different from zero ( $t$ test $p>0.05$ ). Within this continuum, and for descriptive purposes, neurons could be assigned to three different classes: nonadapting neurons (defined as those for which all NSSI values under all conditions were $<0.25$ ), novelty neurons (defined as those for which all NSSI values under all conditions were $>0.5$ ), and partially adapting neurons (those for which NSSI values were $>0.5$ under some but not all conditions) (supplemental Fig. 3, available at www.jneurosci.org as supplemental material). Nonadapting neurons, including those with small negative SSA values, had nearly identical responses to the standard and oddball stimuli under all conditions $(n=51)$, and on average the spike counts in response to the standard and oddball stimuli differed from one another by only $13 \%$; NSSI range, -0.42 to 0.24 ; average, $0.015 \pm 0.09$. Nonadapting neurons made up $34 \%$ of the whole sample. Novelty neurons were defined as those with high SSA under all conditions $(n=28$, NSSI range, $0.56-0.94$; average, $0.76 \pm 0.16$ ), and made up $18 \%$ of the total. For novelty neurons, the response to the oddball stimulus was on average $160 \%$ larger than the response to the standard. The remaining 
neurons (48\%) showed SSA under some conditions but not all ( $n=73$, NSSI range, 0.02-0.66; average, $0.31 \pm 0.12$ ).

As mentioned above, a NSSI value was calculated for each stimulation condition, meaning that each neuron was usually assigned several NSSI values. These NSSI values for each neuron were averaged to obtain the distribution shown in supplemental Fig. 2, available at www.jneurosci. org as supplemental material. We then fitted the distribution with a curve consisting of one-, two-, or three-Gaussian functions. The results show that the three-Gaussian model (see supplemental Fig. 2C, available at www.jneurosci.org as supplemental material) is the one that best characterizes our NSSI distribution $\left(R^{2}=0.981\right)$, and it is also significantly better than the one- or two-Gaussian models. The means of the Gaussian functions that yield the best fit to the NSSI distribution are $0.057,0.384$, and 0.870 , which closely correspond to the average NSSI values of each group following our own classification (nonadapting, 0.015 ; partially adapting, 0.31 ; novelty, $0.76)$. Overall, these data indicate that two thirds of all IC neurons showed at least some degree of SSA.

\section{Relation of SSA to other parameters}

In 171 neurons, we recorded complete FRAs in addition to measuring SSA (Fig. $1 B, C)$. Figure 6, $A$ and $B$, shows $Q_{10}$ and $Q_{40}$ values for those neurons for which we were able to measure a complete FRA. $Q$ values are plotted as a function of the average NSSI for at least three different conditions for each neuron (see above and supplemental material, available at www. jneurosci.org). Although there was a wide range of $Q$ values across neuron-specific SSA index values, there was a distinct trend for nonadapting neurons to have the highest $Q$ values and the largest range of values, followed by partially adapting neurons. Novelty neurons (high SSA values) all had low $Q$ values, indicating broad frequency tuning.

We also compared frequency discrimination ability of IC neurons in the $90 / 10 \%$ and $50 / 50 \%$ conditions, using metrics derived from ROC analysis (Fig. 7), a method that has been widely used in psychophysics and also applied successfully to physiological data (Shackleton et al. 2003; Ulanovsky et al., 2003). We used the area under the curve as an estimate of the percentage of correct frequency identifications, based on the spike count after each stimulus presentation (Ulanovsky et al. 2003). The frequency discriminability was significantly better in the $90 / 10 \%$ condition than in the $50 / 50 \%$ condition for all $\Delta f$ values (Sign test, $p<0.001$ for $\Delta f=0.37,0.10$ or 0.04 ). When considering both $\Delta f$ and the repetition rate, the only combination under which discriminability was not better in the $90 / 10 \%$ condition was when the smallest frequency contrast $\Delta f(0.04)$ was

\section{A $90 / 10 \%$}

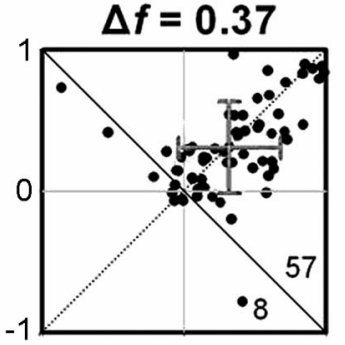

$\Delta f=0.10$
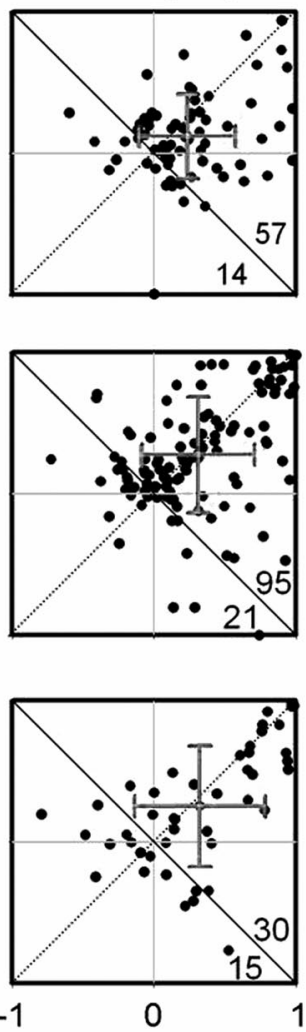

$-1$
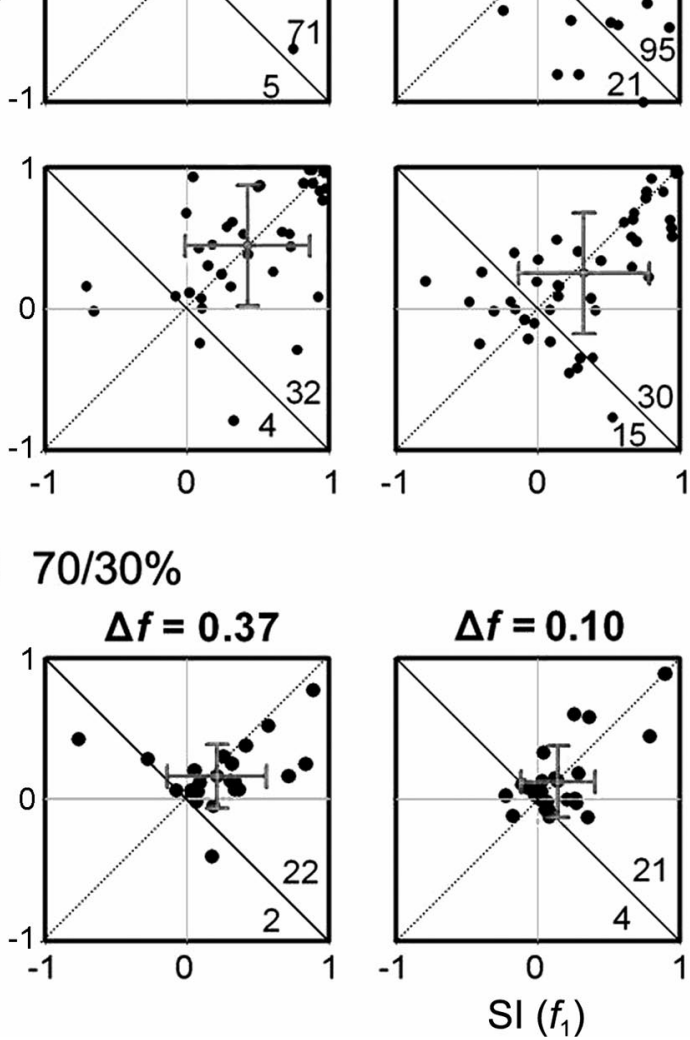

$\Delta f=0.04$

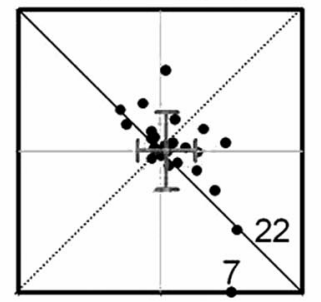

$D$
$\stackrel{D}{D}$
$N$
$\frac{T}{N}$
Figure 5. $\quad \boldsymbol{A}, \boldsymbol{B}$, Scatter plots of the frequency-specific SI at different probabilities $(\boldsymbol{A}, 90 / 10 \% ; \boldsymbol{B}, 70 / 30 \%)$, frequency contrasts (columns), and stimulation rates (rows). Because each neuron was tested using several combinations of parameters, individual neurons may be represented in more than one panel. The numbers on the bottom right indicate the number of dots on each side of the descending diagonal. The gray crosses indicate the mean and SD for each axis.

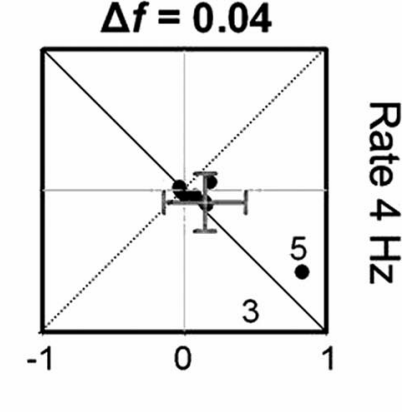

combined with the fastest repetition rate (8/s) (Sign test, $p=$ $0.115)$. For all the other combinations the discriminability in the $90 / 10 \%$ condition was improved relative to the $50 / 50 \%$ condition (Sign test, $p=0.03$ for $\Delta f=0.04,2 / \mathrm{s} ; p=0.019$ for $\Delta f=0.10$, $8 / s ; p<0.001$ for the rest of combinations).

It is noteworthy that in all of the scatterplots shown in Figure $7 A-C$, there are data points in the upper right quadrant. This finding indicates that even for small $\Delta f$ some IC neurons have very high discrimination ability, close to $100 \%$, regardless of the probability of the stimuli presented.

Figure $7 D$ shows how the average discrimination ability of the sampled neurons (gray lines) varies depending on $\Delta f$. Although the discrimination ability increases with larger $\Delta f$, it is always 


\begin{tabular}{|c|c|c|c|}
\hline & \multicolumn{3}{|l|}{ Frequency contrast } \\
\hline & $\Delta f=0.37($ mean $\pm S D)$ & $\Delta f=0.1($ mean $\pm S D)$ & $\Delta f=0.04($ mean $\pm S D)$ \\
\hline \multicolumn{4}{|l|}{$90 / 10 \%$} \\
\hline $2 \mathrm{~Hz}$ & $n=63(0.33 \pm 0.28)$ & $n=73(0.16 \pm 0.24)$ & $n=28(0.06 \pm 0.09)$ \\
\hline Range & -0.09 to 0.93 & -0.53 to 0.92 & -0.06 to 0.39 \\
\hline $4 \mathrm{~Hz}$ & $n=69(0.46 \pm 0.32)$ & $n=122(0.31 \pm 0.35)$ & $n=71(0.17 \pm 0.25)$ \\
\hline Range & -0.1 to 0.99 & -0.43 to 0.98 & -0.22 to 0.88 \\
\hline $8 \mathrm{~Hz}$ & $n=36(0.45 \pm 0.35)$ & $n=43(0.30 \pm 0.36)$ & $n=17(0.17 \pm 0.30)$ \\
\hline Range & -0.16 to 0.97 & -0.28 to 0.97 & -0.08 to 0.76 \\
\hline \multicolumn{4}{|l|}{$70 / 30 \%$} \\
\hline $4 \mathrm{~Hz}$ & $n=24(0.20 \pm 0.19)$ & $n=25(0.12 \pm 0.22)$ & $n=8(0.02 \pm 0.05)$ \\
\hline Range & -0.01 to 0.83 & -0.14 to 0.89 & -0.02 to 0.13 \\
\hline
\end{tabular}
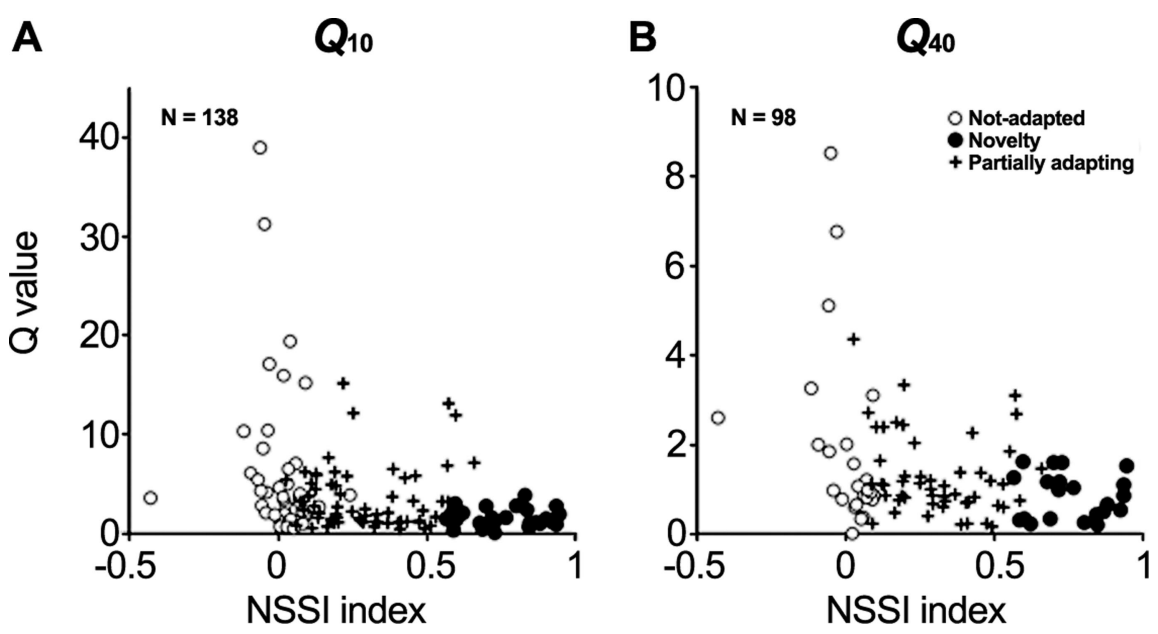

Figure 6. Relationship between individual FRAs and SSA. $\boldsymbol{A}$ and $\boldsymbol{B}$ show the distribution of $Q_{10 \mathrm{~dB}}$ and $Q_{40 \mathrm{~dB}}$ values relative to NSSI. The higher the SSA (NSSI values closer to 1), the more the range of $Q$ values became restricted to small $Q$ values.
(100.6 \pm 4.4 ms; range, 90.82-113.4). Figure $9 B$ is a plot of the difference in the mean first spike latency of the responses to the oddball and the standard for each frequency as a function of the NSSI. Histogram distributions of latencies to the standard and oddball are shown in supplemental Figure 4, available at www.jneurosci. org as supplemental material. For neurons with large NSSI values, the latency difference was generally larger, reflecting a shorter latency in response to the oddball. However, we also performed an additional analysis whereby we averaged all latencies from the different conditions tested for each neuron and plotted average latency as a function of NSSI (Fig. 9). There was a wide range of average latency across all values of NSSI, but the shortest latencies $(<15 \mathrm{~ms})$ were all found at NSSI values $<0.5$. This finding could reflect the fact that latencies in response to the standard smaller in the 50/50\% condition (dashed lines) than in the $90 /$ $10 \%$ condition (solid lines). This is still true when analyzing only the most sensitive $10 \%$ of neurons in the sample (black lines), but now the overall values are close to $100 \%$ [compare our data with AC data in Ulanovsky et al. (2003), their Fig. 3]. The difference in discrimination ability across conditions is also related to the NSSI of the neuron. Neurons with high NSSI show a larger increment in discriminability going from the $50 / 50 \%$ to the $90 / 10 \%$ condition. The difference between the $90 / 10 \%$ and $50 / 50 \%$ conditions is positively correlated with NSSI $\left(R^{2}=0.45\right)$. Although this correlation is not very strong, it nevertheless indicates a trend.

Many neurons also showed "latency adaptation" (Fig. 8A), whereby the average first spike latency of the response evoked by a given frequency was significantly ( $t$ test, $p<0.001$ ) shorter when that frequency was presented as the oddball stimulus (26.1 $\pm 13.2 \mathrm{~ms}$; range, 7.5-72) than when it was presented as the standard (29.6 $\pm 13.2 \mathrm{~ms}$; range, 7.3-74.5). Figure $8 \mathrm{~A}$ shows plots of first-spike latency in response to the standard stimulus versus latency in response to the oddball. The majority of values lie on or above the bisecting diagonal line, indicating that most neurons responded to the oddball with the same latency or a shorter latency than to the standard. There was also a cluster of a few neurons with latencies longer than $90 \mathrm{~ms}$ that correspond to neurons that responded to the offset of the stimulus. In this case also, latency of the response to a frequency presented as the oddball stimulus (97.2 $\pm 3.2 \mathrm{~ms}$; range, 90-102) was shorter than the latency to the same frequency presented as the standard stimulus stimulus were longer for those neurons showing strong SSA, or it could indicate that a certain amount of processing time is required for these neurons to respond regardless of stimulus conditions.

\section{Time course of adaptation}

To visualize the time course of adaptation in the population of IC neurons, we examined how responses develop over consecutive trials. Figure $8 C$ shows the population average response as a function of trial number within the train for both standard and oddball stimuli. At all frequency contrast values, SSA in response to the standard occurred rapidly, reaching a maximum within the first 20-25 trials. The response to the oddball showed little or no adaptation over the course of a train, although it was considerably more variable, probably because of the smaller number of presentations of the oddball stimulus. This time scale looks quite similar to the one presented by Malone and Semple (2001) for monaural and binaural conditioning stimuli, which were approximately equivalent to our $90 \%$ standard paradigm. This figure also shows that the oddball stimuli evoke stronger responses than the standard stimuli, particularly at the larger frequency contrast values.

\section{SSA is ubiquitous in all IC subdivisions}

Figure 10 shows two collapsed three-dimensional reconstructions of sections through the IC to indicate the locations of those neurons at recording sites that were histologically identified $(n=$ 

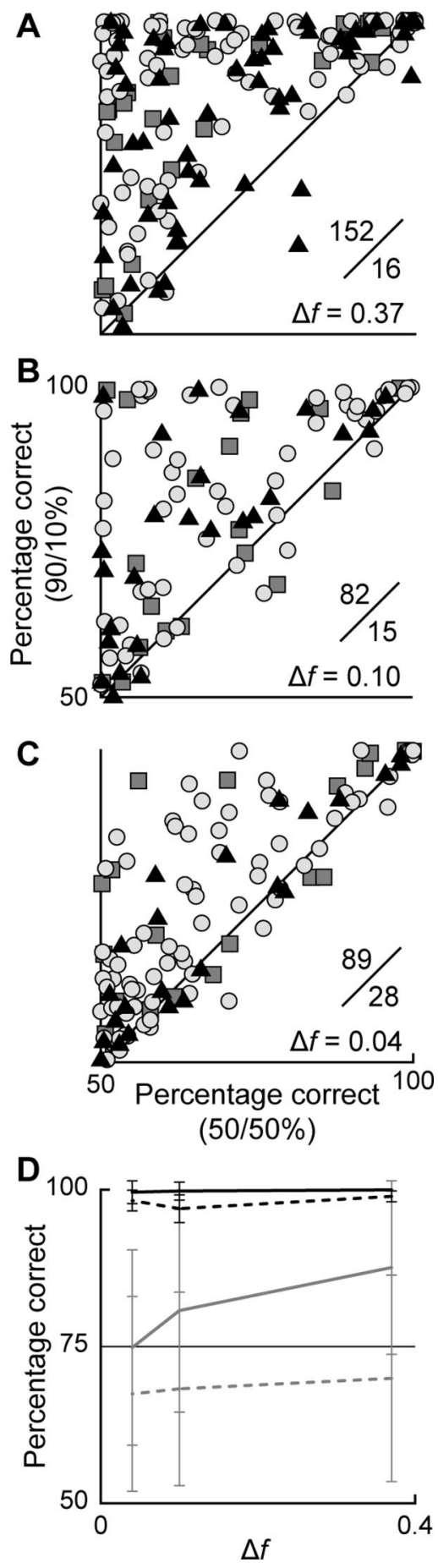

Figure 7. A-C, Scatter plots based on ROC curve analysis comparing frequency discrimination by IC neurons in the $90 / 10 \%$ condition versus the $50 / 50 \%$ condition, for $\Delta f=0.37,0.10$, and 0.04 . Each dot represents one neuron, and the symbols indicate the repetition rate: triangles, $2 / s$; circles, $4 / s ;$ squares, $8 / 5$. The number of data points above and below the diagonal is shown in an inset; dots above the diagonal mean better discriminability for the $90 / 10 \%$ condition than for $50 / 50 \%$. D, Variation of the average discriminability (mean \pm SD) depending on $\Delta$ f. Solid lines, 90/10\%; dashed lines, 50/50\%; gray lines, data including all neurons in the sample; black lines, data taken from only the $10 \%$ most sensitive neurons.

141). Most neurons were recorded from the rostral and lateral portions of the IC, with 41 neurons located in the central nucleus of the inferior colliculus (CNIC). All three response types (nonadapting, partially adapting, and novelty neurons) were distributed throughout the entire rostrocaudal, dorsoventral, and me- diolateral axes of the IC and were not confined to any particular subdivision (Fig. 10). However, novelty neurons, i.e., those with a high level of SSA, were more prevalent in the lateral and dorsal part of the inferior colliculus. NSSI values were, on average, significantly ( $t$ test, $p<0.001)$ lower in the CNIC $(0.17 \pm 0.27)$ than in the cortical regions of the IC $(0.3 \pm 0.32)$, but neurons that exhibited SSA clearly were also present in the central nucleus. These data suggest that SSA is ubiquitous throughout the IC. Although we chose frequency as a convenient parameter to vary, and to allow a direct comparison with the results described by Ulanovsky et al. (2003, 2004), it is very likely that changing other parameters such as sound level or duration would also cause release from SSA, as has been suggested in our previous study (Pérez-González et al., 2005).

\section{Discussion}

This study demonstrates that a high percentage of neurons throughout the IC display some degree of SSA. In this respect, IC neurons resemble cortical neurons, most of which also show some degree of SSA (Ulanovsky et al., 2003). Different forms of SSA have been shown in the auditory system, particularly at the cortical level (Condon and Weinberger, 1991; Malone et al., 2002; Sanes et al., 1998; Ulanovsky et al., 2003), but no previous studies in mammals have used the oddball paradigm to observe SSA at the midbrain. One recent study in the barn owl (Reches and Gutfreund, 2008) reported SSA in the avian equivalent of the IC, suggesting that it is a phenomenon common to all vertebrates. Although neurons with a high degree of SSA appear to be most common in the dorsal and external cortices of the IC (PérezGonzález et al., 2005), neurons with SSA are distributed throughout the entire IC, including the central nucleus. There is a gradient in the amount of SSA expressed by IC neurons, with those in the dorsal, lateral, and rostral IC exhibiting the most pronounced form (Pérez-González et al., 2005).

IC neurons that express SSA respond with a variety of discharge patterns. However, those neurons that have a high degree of SSA and are selective for novel stimuli are all onset responders (Pérez-González et al., 2005). There is a distinct relationship between the SSA index and the frequency tuning of neurons; i.e., neurons with the highest SSA indices were the most broadly tuned. It is logical that those neurons that are best suited to adapt to repeated sounds and respond to novel sounds should receive input over a broad frequency range. In the rat, neurons in the dorsal and rostral IC have the largest dendritic arbors (Malmierca et al., 1993, 1995), and thus, are in a position to integrate inputs across many frequencies.

The extent to which IC neurons express SSA depends on stimulus repetition rate, the amount of frequency contrast between standard and oddball stimuli, and the probability of the oddball stimulus occurring. SSA was greatest at the largest frequency contrast, the lowest probability of the oddball stimulus, and a repetition rate of $4 / \mathrm{s}$. At a rate of $8 / \mathrm{s}$, many neurons ceased responding entirely, suggesting that, in addition to SSA, they experienced temporal masking (Faure et al., 2003). Presumably at a repetition rate of $4 / \mathrm{s}$ stimulus duty cycle is sufficient to create SSA, but the interstimulus interval is long enough so that the effects of forward and backward masking do not reduce the responsiveness of the neuron significantly. These observations suggest that SSA may be interactive with temporal masking in IC neurons.

\section{Comparison of SSA in midbrain and cortical neurons}

The present study demonstrates that there are both similarities and differences in the dynamics of adaptation between the IC and 
A

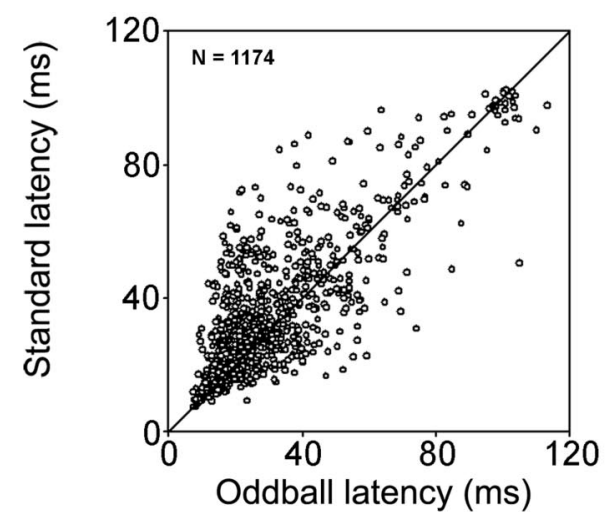

B

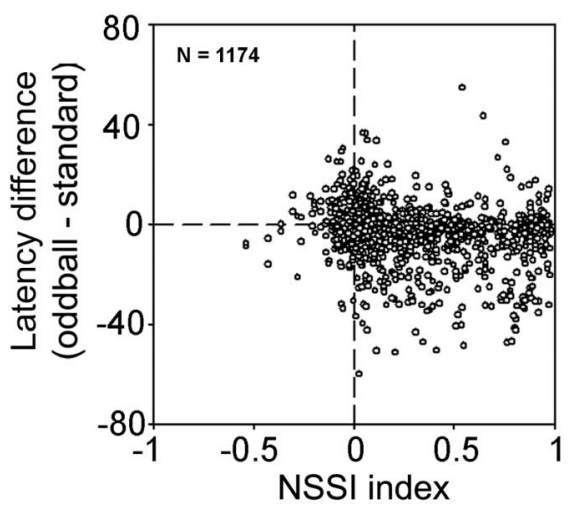

C

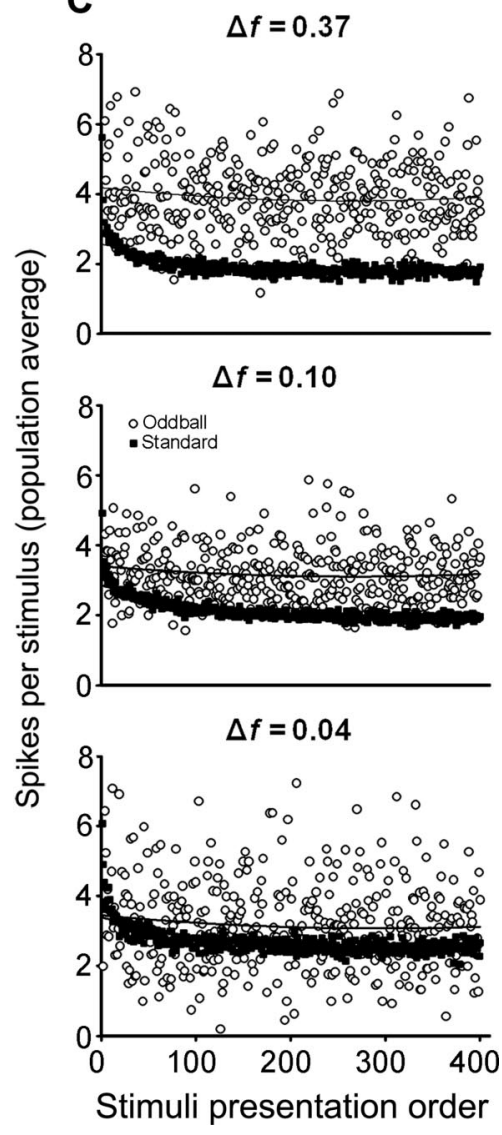

Figure 8. $\quad \boldsymbol{A}$, Comparison of mean first spike latency of responses to a given tone when it was presented as the standard stimulus and when it was presented as the oddball. Although there are data points on both sides of the 1:1 line, the majority of neurons have a standard latency that is longer than the oddball latency. $\boldsymbol{B}$, When the difference between the standard and the oddball latencies is plotted as a function of the neuron-specific SSA index, it becomes apparent that in neurons with a high degree of SSA, the standard latency tends to be longer than the oddball latency. $\boldsymbol{C}$, Time course of adaptation. The average number of spikes per stimulus in the sample is plotted against the order in which that stimulus occurred. Note how the response decays rapidly in the case of the standard stimuli resulting from adaptation, but not for the oddball stimuli. The higher variability in the responses to the oddball is probably caused by the smaller number of stimulus presentations, compared with the standard. Also note that the response to the oddball stimuli is stronger at a frequency contrast of 0.37 , but it approaches the response to the standard as the frequency contrast is reduced.

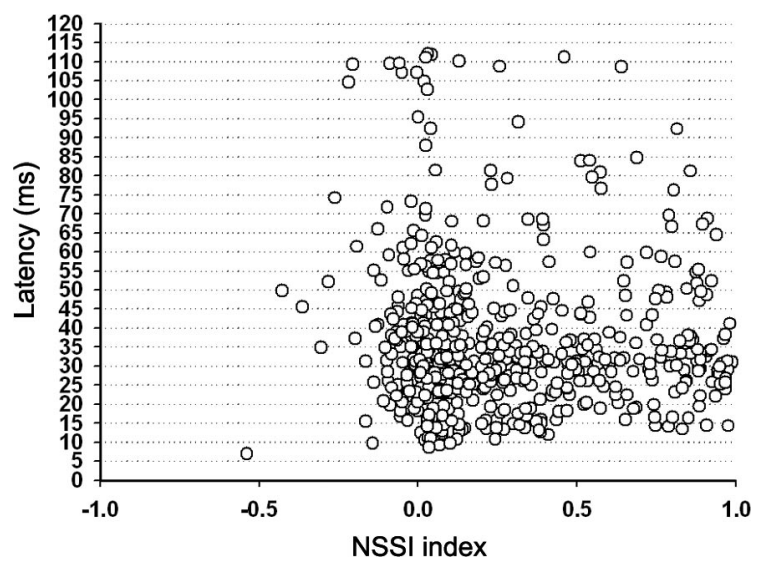

Figure 9. Distribution of the average first-spike latency for both standard and oddball stimuli as a function of NSSI. Note that there is no correlation between NSSI and latency.

AC (Ulanovsky et al., 2003). Before we discuss these issues in a functional context we must highlight a major methodological difference between the study of Ulanovsky and colleagues (AC) and ours (IC). The data obtained in the IC is based on relatively

fast repetition rates $(2,4$, and $8 \mathrm{~Hz})$, compared with that of the AC $(0.25-3 \mathrm{~Hz})$.

SSA in the IC resembles SSA in the AC in that the difference between the responses to the oddball and standard stimuli is positively correlated with the amount of frequency contrast and the stimulus repetition rate (up to $4 / \mathrm{s}$ ). Moreover, it is negatively correlated with the probability of occurrence of the oddball stimulus. In both the AC and IC there appears to be a continuum in the amount of frequency contrast to which neurons are sensitive.

Another important similarity between SSA in AC and IC is the rapid time course of adaptation to the standard stimulus. Across all IC neurons, there was a rapid response decay over the first 10 presentations followed by a slower decay that reached a maximum within 25 presentations. There was no significant adaptation to the oddball stimulus over the course of all presentations, possibly because of the fact that it occurred at a slower repetition rate, or possibly because the intervening stimuli caused a release from adaptation. A further important and unexpected similarity between the SSA in the IC and AC is that IC neurons also show hyperacuity for very small $\Delta f$, as small as 0.057 octaves (compare Fig. 7C).

SSA in the IC differs from that in the AC because (1) very few AC neurons respond to the standard and oddball stimuli with different latencies (Ulanovsky et al., 2004); in contrast, most IC neurons responded to a given frequency when presented as the oddball stimulus with a shorter latency (Fig. 8). The data are consistent with the idea that the neuron is driven more effectively by the oddball stimulus than by the standard. (2) The biggest difference between the response to the oddball and standard occurs during the sustained portion of the response in AC neurons, whereas in the IC it occurred during the onset portion. (3) In the AC, there is no correlation between SSA and breadth of frequency tuning, whereas these measures are strongly correlated in IC neurons.

The similarities and differences detailed above could be caused by several factors, including the anesthetic used (halothane in the cat vs urethane in the rat) and/or the animal model (cat vs rat). For example, the auditory forebrain of the rat and cat are anatomically different (Winer, 2006; Winer and Lee, 2007). Electrophysiological studies have demonstrated species differences in the cortical processing of sound parameters such as FM sweeps (Nelken and Versnel, 2000). However, the present study shows that the differences seen are not likely caused by experimental design and methodology because, except for the faster repetition rate used in our study, the methods were very similar. The differences observed may therefore reflect different processing mechanisms at the midbrain and cortex. 

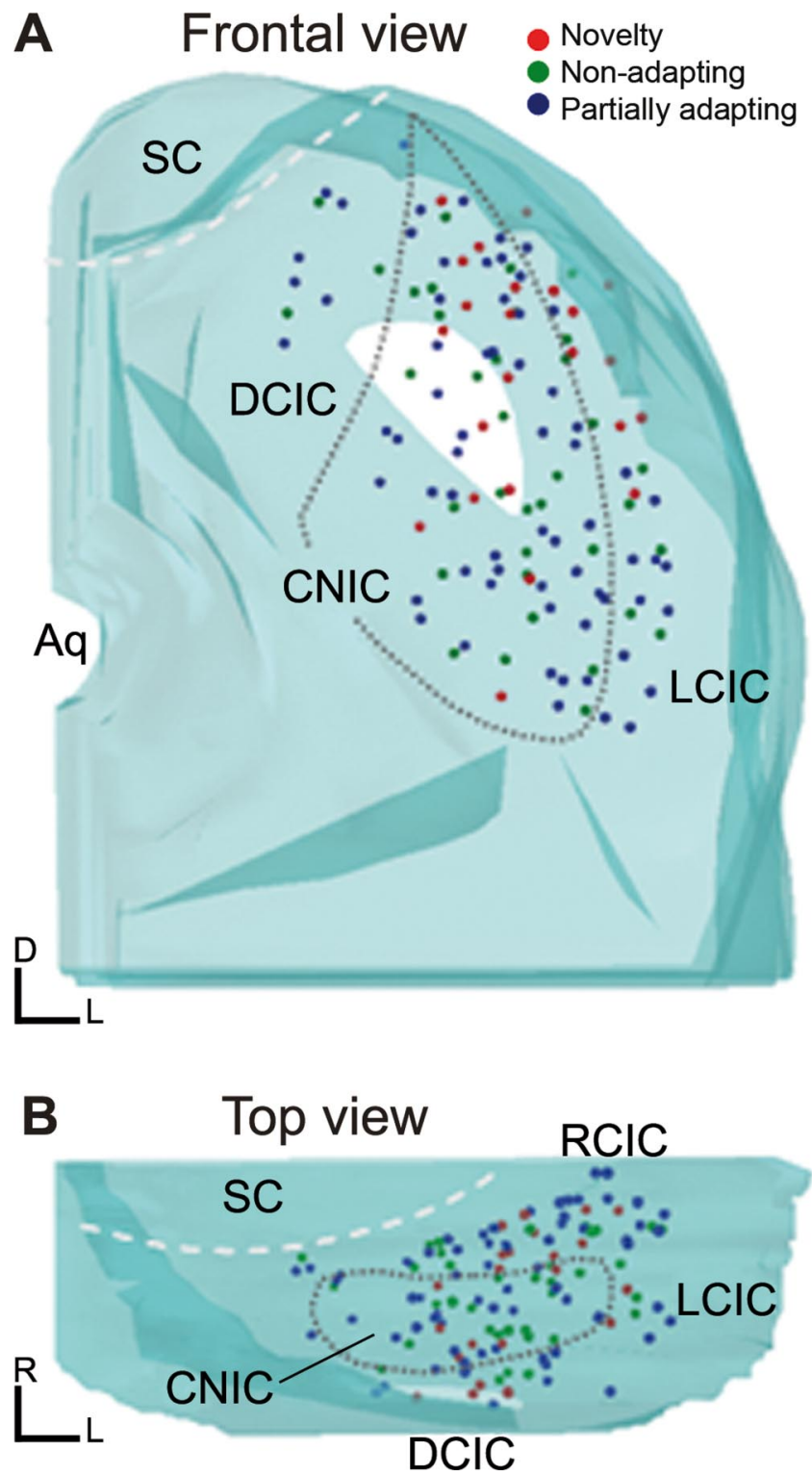

Figure 10. $\quad \boldsymbol{A}, \boldsymbol{B}$, Computer-assisted three-dimensional reconstructions of the locations of neurons that were histologically localized, seen in a frontal projection $(\boldsymbol{A})$ and in a horizontal projection $(\boldsymbol{B})$, rotated $90^{\circ}$ from the projection seen in $\boldsymbol{A}$. Neurons showing the entire range of SSA were found in all subdivisions of the $I C$, although a higher proportion of the neurons with the highest degree of SSA were located in the dorsal and rostral parts of the IC. Aq, Aqueduct; DCIC, dorsal cortex of the inferior colliculus; LCIC, lateral cortex of the inferior colliculus; RCIC, rostral cortex of the inferior colliculus; SC, superior colliculus.

\section{Is SSA a bottom-up or a top-down process?}

An important question is whether or not SSA in the IC is created de novo at the midbrain and transmitted from there to the cortex, or whether it is caused by descending projections from the cortex. It is well known that the $\mathrm{AC}$ has a dense projection to the cortices of the IC in the rat (Saldaña et al., 1996; Winer et al., 1998; Bajo and Moore, 2005; Bajo et al., 2007; Malmierca and Ryugo, 2009), so it is possible that SSA in those regions could reflect, or be enhanced by, descending cortical input. Ulanovsky et al. (2003) showed that the largest difference between the responses to the standard and oddball stimuli occurs during the steady state portion of the response, suggesting some intracortical processing is responsible for SSA. In contrast, we observed that for most IC neurons, the difference between the response to the standard and oddball was maximal during the onset portion of the response. These findings argue against the idea that SSA in the IC is imposed by descending projections from the AC. There is contradictory evidence regarding neurons in the MGB (Kraus et al., 1994; Ulanovsky et al., 2003; Antunes et al., 2009), which receive a much stronger direct descending projection than the IC (Winer et al., 1998, 2001; Malmierca and Ryugo, 2009), but receive the bulk of their ascending inputs from the IC (Oliver et al., 1999; Malmierca et al., 2002). The most likely scenario is that SSA is generated at the IC through intrinsic circuitry (see above), but modulated by descending cortical input so that the system includes both bottom-up and top-down processing. The latency analysis shown in Figure 9 indicates that those IC neurons with the strongest SSA do not necessarily possesses the longest latencies, but that the shortest latencies of these neurons are longer than the shortest latencies of nonadapting IC neurons. Thus, there is some support for the idea that additional processing time is required for a descending cortical modulation to take effect, at least in a subset of neurons.

In conclusion, the present data demonstrate that SSA is a single-neuron level phenomenon that may provide one of the mechanisms underlying novelty detection. Previous studies (Ulanovsky et al., 2003) have linked the emergence of SSA at the cortical level with auditory memory (Nelken et al., 2003). The existence of SSA at both the midbrain and cortex strongly suggests that there are two separate SSA mechanisms, one that is preattentive and operates subcortically [present study, Galbraith et al. (2004), Rinne et al. (2008)], affecting primarily the onset portion of the response, and another that operates at the cortical level where it mainly affects the sustained portion of the response (Ulanovsky et al., 2003). Whether they are complementary, hierarchical, interactive, or redundant (Chechik et al., 2006) is a matter for further studies. If SSA is part of the neural process underlying $M M N$, then the current data show that MMN, although not directly related to action potential activity, may be generated through several hierarchically organized steps and is not merely the result of processing that begins at the cerebral cortex.

\section{References}

Antunes FM, Covey E, Malmierca MS (2009) Is there stimulus-specific adaptation in the auditory thalamus? In: Advances in auditory physiology, psychophysics and models (Lopez-Poveda EA, Palmer AR, Meddis R, eds). New York: Springer, in press.

Bajo VM, Moore DR (2005) Descending projections from the auditory cortex to the inferior colliculus in the gerbil, Meriones unguiculatus. J Comp Neurol 486:101-116.

Bajo VM, Nodal FR, Bizley JK, Moore DR, King AJ (2007) The ferret auditory cortex: descending projections to the inferior colliculus. Cereb Cortex 17:475-491.

Chechik G, Anderson MJ, Bar-Yosef O, Young ED, Tishby N, Nelken I (2006) Reduction of information redundancy in the ascending auditory pathway. Neuron 51:359-368.

Condon CD, Weinberger NM (1991) Habituation produces frequencyspecific plasticity of receptive fields in the auditory cortex. Behav Neurosci 105:416-430.

Csépe V, Karmos G, Molnár M (1987a) Effects of signal probability on sensory evoked potentials in cats. Int J Neurosci 33:61-71.

Csépe V, Karmos G, Molnár M (1987b) Evoked potential correlates of stimulus deviance during wakefulness and sleep in cat-animal model of mismatch negativity. Electroencephalogr Clin Neurophysiol 66:571-578.

Deouell LY (2007) The frontal generator of mismatch negativity revisited. J Psychophysiol 21:188-203.

Faure PA, Fremouw T, Casseday JH, Covey E (2003) Temporal masking reveals properties of sound-evoked inhibition in duration-tuned neurons of the inferior colliculus. J Neurosci 23:3052-3065.

Fritz JB, Elhilali M, David SV, Shamma SA (2007) Auditory attention-focusing the searchlight on sound. Curr Opin Neurobiol 17:437-455. 
Galbraith GC, Gutterson RP, Levy DS, Mussey JL, Sabatasso FA, Wasserman RI. (2004) Correlated brain stem and cortical evoked responses to auditory tone change. Neuroreport 15:2613-2616.

Hara K, Harris RA (2002) The anesthetic mechanism of urethane: the effects on neurotransmitter-gated ion channels. Anesth Analg 94:313-318, table of contents.

Hernández O, Espinosa N, Pérez-González D, Malmierca MS (2005) The inferior colliculus of the rat: a quantitative analysis of monaural frequency response areas. Neuroscience 132:203-217.

Kraus N, McGee T, Littman T, Nicol T, King C (1994) Nonprimary auditory thalamic representation of acoustic change. J Neurophysiol 72:1270-1277.

LeBeau FE, Malmierca MS, Rees A (2001) Iontophoresis in vivo demonstrates a key role for GABA(A) and glycinergic inhibition in shaping frequency response areas in the inferior colliculus of guinea pig. J Neurosci 21:7303-7312.

Loftus WC, Malmierca MS, Bishop DC, Oliver DL (2008) The cytoarchitecture of the inferior colliculus revisited: a common organization of the lateral cortex in rat and cat. Neuroscience 154:196-205.

Malmierca MS (2003) The structure and physiology of the rat auditory system: an overview. Int Rev Neurobiol 56:147-211.

Malmierca MS, Ryugo DK (2009) Cortical descending projections to auditory midbrain and brainstem. In: The auditory cortex (Winer JA, Schreiner CE, eds), New York: Springer.

Malmierca MS, Blackstad TW, Osen KK, Karagülle T, Molowny RL (1993) The central nucleus of the inferior colliculus in rat: a Golgi and computer reconstruction study of neuronal and laminar structure. J Comp Neurol 333:1-27.

Malmierca MS, Seip KL, Osen KK (1995) Morphological classification and identification of neurons in the inferior colliculus: a multivariate analysis. Anat Embryol (Berl) 191:343-350.

Malmierca MS, Merchán MA, Henkel CK, Oliver DL (2002) Direct projections from cochlear nuclear complex to auditory thalamus in the rat. J Neurosci 22:10891-10897.

Malmierca MS, Hernández O, Falconi A, Lopez-Poveda EA, Merchán M, Rees A (2003) The commissure of the inferior colliculus shapes frequency response areas in rat: an in vivo study using reversible blockade with microinjection of kynurenic acid. Exp Brain Res 153:522-529.

Malmierca MS, Hernández O, Rees A (2005a) Intercollicular commissural projections modulate neuronal responses in the inferior colliculus. Eur J Neurosci 21:2701-2710.

Malmierca MS, Saint Marie RL, Merchan MA, Oliver DL (2005b) Laminar inputs from dorsal cochlear nucleus and ventral cochlear nucleus to the central nucleus of the inferior colliculus: two patterns of convergence. Neuroscience 136:883-894.

Malmierca MS, Cristaudo S, Pérez-González D, Covey E (2007) Novelty responses in the rat auditory midbrain: evidence from an oddball paradigm. ARO Abstr 212.

Malmierca MS, Izquierdo MA, Cristaudo S, Hernández O, Pérez-González D, Covey E, Oliver DL (2008) A discontinuous tonotopic organization in the inferior colliculus of the rat. J Neurosci 28:4767-4776.

Malone BJ, Semple MN (2001) Effects of auditory stimulus context on the representation of frequency in the gerbil inferior colliculus. J Neurophysiol 86:1113-1130.

Malone BJ, Scott BH, Semple MN (2002) Context-dependent adaptive coding of interaural phase disparity in the auditory cortex of awake macaques. J Neurosci 22:4625-4638.

Merrill EG, Ainsworth A (1972) Glass-coated platinum-plated tungsten microelectrodes. Med and Biol Eng 10:662-672.

Näätänen R (1992) Attention and brain function. Hillsdale, NJ: Lawrence Erlbaum.
Näätänen R, Michie PT (1979) Early selective-attention effects on the evoked potential: a critical review and reinterpretation. Biol Psychol 8:81-136

Näätänen R, Gaillard AW, Mantysalo S (1978) Early selective-attention effect on evoked potential reinterpreted. Acta Psychol (Amst) 42:313-329.

Nelken I, Ulanovsky N (2007) Mismatch negativity and stimulus-specific adaptation in animal models. J of Psycophysiol 214-223.

Nelken I, Versnel H (2000) Responses to linear and logarithmic frequencymodulated sweeps in ferret primary auditory cortex. Eur J Neurosci 12:549-562.

Nelken I, Fishbach A, Las L, Ulanovsky N, Farkas D (2003) Primary auditory cortex of cats: feature detection or something else? Biol Cybern 89:397-406.

Oliver DL, Ostapoff EM, Beckius GE (1999) Direct innervation of identified tectothalamic neurons in the inferior colliculus by axons from the cochlear nucleus. Neuroscience 93:643-658.

Paxinos G, Watson C (2005) The Rat brain in stereotaxic coordinates. Burlington: Elsevier-Academic.

Pérez-González D, Malmierca MS, Covey E (2005) Novelty detector neurons in the mammalian auditory midbrain. Eur J Neurosci 22:2879-2885.

Pérez-González D, Malmierca MS, Moore JM, Hernández O, Covey E (2006) Duration selective neurons in the inferior colliculus of the rat: topographic distribution and relation of duration sensitivity to other response properties. J Neurophysiol 95:823-836.

Reches A, Gutfreund Y (2008) Stimulus-specific adaptations in the gaze control system of the barn owl. J Neurosci 28:1523-1533.

Rees (1990) A A close-field sound system for auditory neurophysiology. J of Physiol 430:2.

Rees A, Sarbaz A, Malmierca MS, Le Beau FE (1997) Regularity of firing of neurons in the inferior colliculus. J Neurophysiol 77:2945-2965.

Rinne T, Balk MH, Koistinen S, Autti T, Alho K, Sams M (2008) Auditory selective attention modulates activation of human inferior colliculus. J Neurophysiol 100:3323-3327.

Saldaña E, Feliciano M, Mugnaini E (1996) Distribution of descending projections from primary auditory neocortex to inferior colliculus mimics the topography of intracollicular projections. J Comp Neurol 371:15-40.

Sanes DH, Malone BJ, Semple MN (1998) Role of synaptic inhibition in processing of dynamic binaural level stimuli. J Neurosci 18:794-803.

Schönwiesner M, Novitski N, Pakarinen S, Carlson S, Tervaniemi M, Näätänen R (2007) Heschl's gyrus, posterior superior temporal gyrus, and mid-ventrolateral prefrontal cortex have different roles in the detection of acoustic changes. J Neurophysiol 97:2075-2082.

Shackleton TM, Skottun BC, Arnott RH, Palmer AR (2003) Interaural time difference discrimination thresholds for single neurons in the inferior colliculus of Guinea pigs. J Neurosci 23:716-724.

Tiitinen H, May P, Reinikainen K, Näätänen R (1994) Attentive novelty detection in humans is governed by pre-attentive sensory memory. $\mathrm{Na}$ ture 372:90-92.

Ulanovsky N, Las L, Farkas D, Nelken I (2004) Multiple time scales of adaptation in auditory cortex neurons. J Neurosci 24:10440-10453.

Ulanovsky N, Las L, Nelken I (2003) Processing of low-probability sounds by cortical neurons. Nat Neurosci 6:391-398.

Winer JA (2006) Decoding the auditory corticofugal systems. Hear Res 212:1-8.

Winer JA, Lee CC (2007) The distributed auditory cortex. Hear Res 229:3-13.

Winer JA, Larue DT, Diehl JJ, Hefti BJ (1998) Auditory cortical projections to the cat inferior colliculus. J Comp Neurol 400:147-174.

Winer JA, Diehl JJ, Larue DT (2001) Projections of auditory cortex to the medial geniculate body of the cat. J Comp Neurol 430:27-55. 